\title{
Icariin ameliorates the cognitive function in an epilepsy neonatal rat model by blocking the GluR2/ERK I/II pathway
}

\author{
Ying Guo ${ }^{1}$, Yuxiang $\mathrm{Cai}^{2}$, Xiaoge Zhang ${ }^{1}$ \\ ${ }^{1}$ Department of Neurology, Northwest Women's and Children's Hospital, Xi'an, Shaanxi, China, ${ }^{2}$ Department of Emergency, \\ Xi'an Children's Hospital, Xi'an, Shaanxi, China
}

\begin{abstract}
The present study was performed to evaluate the protective effects of icariin on cognitive function in a hypoxiainduced neonatal epilepsy rat model. Neonatal epilepsy was induced in rat pups on postnatal day (PD) 20 by induction of hypoxia for 15 minutes. Rats were treated intraperitoneally with icariin at $75 \mathrm{mg} / \mathrm{kg} 1$ hour before the induction of hypoxia. The effects of icariin were examined by estimating seizure stage, cognitive function and parameters of electroencephalography $(E E G)$ in this neonatal epilepsy rat model. Parameters of oxidative stress and expression of proteins were examined in the brain tissue of the neonatal epilepsy rat model by histopathological study and Western blotting analysis, respectively. The results of this study suggest that treatment with icariin ameliorates the changes in seizure stage, number of seizures and parameters of EEG in hypoxia-induced neonatal epilepsy rats. Oxidative stress and apoptosis were decreased in the brain tissue of the icariin treatment group compared to the hypoxia group. Moreover, treatment with icariin ameliorated the altered expression of glutamate ionotropic receptor AMPA type subunit 2 (GluR2) and extracellular receptor kinase (ERK I/II) proteins in the brain tissue of hypoxia-induced epilepsy rats. Histopathological study also showed that icariin treatment improved the histopathology of brain tissue of hypoxia-induced epilepsy rats. In conclusion, the results of the present study suggest that icariin protects against neuronal injury and improves cognitive function in hypoxia-induced neonatal epilepsy rats by modulating the GluR2/ERK I/II pathway.
\end{abstract}

Key words: cognitive function, epilepsy, icariin, hypoxia, neonatal.

\section{Introduction}

Epilepsy is a neurological disease characterised by uncontrolled firing of neurons [17]. There are several types of epilepsy, and neonatal epilepsy occurs due to hypoxic-ischemic encephalopathy and perinatal asphyxia [6]. The rate of the occurrence of seizures is higher in the neonatal period, but conventional anti-epileptic therapies are not effective against neonatal seizures. Neonatal seizures increase the rate of development of neurocognitive dysfunction and epilepsy [9]. In the early postnatal period, the level of GABA receptor expression is lower and that of excitatory ionotropic glutamate receptor is higher than at later stages [1]. $\alpha$-Amino-3-hydroxy-5-methyl-4-isoxazolepropionic acid (AMPA) receptor antagonists were reported to be effective against chronic hyperexcitability and hypoxia-induced seizures in immature rats, but NMDA receptor antagonists did not 
show promising effects [18]. Reduced glutamate receptor 2 (GluR2) expression in the neocortex and hippocampus was reported to be responsible for increased levels of $\mathrm{Ca}^{2+}$-permeable AMPARs [10]. Expression of GluR2 was suggested to be reduced in the hippocampus region in hypoxia-induced seizures, leading to enhanced AMPAR-mediated hippocampal epileptiform activity [16].

Herbal medicine has shown potential effects against the management of several disorders, including neurological disorders. Icariin is a flavonoid and the main active compound isolated from plants belonging to the genus Epimedium (Berberidaceae) [19]. In China, Epimedium is traditionally used for the management of reserve numbness, sexual poten$c y$, and muscle and bone strength. Icariin has been reported to show a number of activities, including cardioprotective, aphrodisiac, immunomodulatory, neuroprotective, anti-inflammatory and anti-oxidant effects $[4,12,15,21,24]$. There is evidence in the literature that icariin can cross the blood-brain barrier and protect against neuronal injury [22]. Icariin shows promising anti-inflammatory and immunomodulatory properties via the regulation of interleukins, nuclear factor $\kappa B(N F-\kappa B)$, c-Jun N-terminal kinase (JNK), glucocorticoids and natural killer (NK) cell activity [5]. The present study was performed to evaluate the beneficial effects of icariin against neonatal epilepsy.

\section{Material and methods \\ Animals}

The study was performed in male Long-Evans rats on postnatal day (PD) 20. All pups were maintained under controlled conditions of 12-hour light/dark cycle, $25 \pm 3^{\circ} \mathrm{C}$ and $60 \pm 5 \%$ humidity. All experimental protocols were approved by the Institutional Animal Ethical Committee of Northwest Women's and Children's Hospital, Xi'an, China (IAEC/NWCH/2018/09).

\section{Hypoxia-induced seizures}

Seizures were induced in the PD20 rat pups by induction of hypoxia for 15 minutes as reported previously [11]. Briefly, all pups were exposed to an oxygen concentration of $7 \%$ for 8 minutes, $5 \%$ for 6 minutes and $4 \%$ for 1 minute for a total period of 15 minutes of hypoxia and the numbers of clonic-tonic seizures were determined. Animals were returned to the cage within 2 hours and the body temperature of each animal was controlled within the range of $32-34^{\circ} \mathrm{C}$.
The animals were divided into three different groups: normal group, hypoxia group treated with saline solution, and the icariin group that received icariin at a dose of $75 \mathrm{mg} / \mathrm{kg}$, i.p. 1 hour before the induction of hypoxia.

\section{Assessment of behavioural changes}

Behavioural changes were observed by determining the changes in the seizure stage using the Racine scale: stage 0 - no response, stage 1 - hyperactivity, stage 2 - clonus and myoclonic jerks of the head, stage 3 - unilateral forelimb clonus, stage 4 - rearing with bilateral forelimb clonus, stage 5 - loss of righting reflex in clonic-tonic seizure.

\section{Assessment of cognitive function}

The Morris water maze test was used to assess cognitive function as reported previously [20]. Briefly, the platform was hidden during the first 4 days of the study trial and the protocol probe trial was performed on the fifth day. Several parameters were analysed according to the percentage of time spent in the target quadrant, platform crossing frequency and escape latency.

\section{Assessment of electroencephalography recordings}

In all animals, electrodes were placed subcutaneously in the dorsal scalp, with two wires from the left and right regions of the parietal lobe and one wire at the midline over the olfactory bulb. Electroencephalography (EEG) was performed using a Stellate Harmonie EEG Unit (version 6.0; Stellate, Montréal, QC, Canada).

\section{Measurement of oxidative stress}

Riboflavin-sensitized photodynamic spectrophotometry was used to assess the activity of superoxide dismutase (SOD), and the absorbance was measured at $460 \mathrm{~nm}$. The method reported by Ohkawa was used to determine the level of lipid peroxidation in brain tissues. The malondialdehyde (MDA) level was determined in brain tissue at a wavelength of $523 \mathrm{~nm}$.

\section{Western blotting}

Caspase-3, Bcl-2, Bax, GluR2, ERK I/II and phosphorylated ERK I/II (p-ERK I/II) expression were 
assessed in isolated brain tissues by Western blotting. The protein contents in the tissue homogenates were determined using a bicinchonic acid (BCA) assay kit, and proteins were separated by $10 \%$ sodium dodecyl sulphate-polyacrylamide gel electrophoresis. The separated proteins were transferred onto membranes, which were then blocked with 5\% blocking reagent. The membranes were then incubated with primary antibodies against caspase-3, Bcl-2, Bax, GluR2, ERK I/II, p-ERK I/II and $\beta$-actin overnight at $4^{\circ} \mathrm{C}$. After washing with phosphate-buffered saline, the membranes were incubated with horseradish peroxidase-conjugated goat secondary antibodies and protein expression was detected using a chemiluminescence kit.

\section{Nissl staining}

All animals were sacrificed and the brains were dissected out and fixed for 1 day in 4\% paraformaldehyde. Brain tissues were dehydrated with alcohol and embedded in paraffin. The paraffin-embedded brain tissue was cut into sections $5-\mu \mathrm{m}$ thick, which were subjected to Nissl staining with $1 \%$ thionin. Neuronal cells were counted under a light microscope.

\section{Statistical analysis}

All data are expressed as means \pm standard error of the mean (SEM; $n=8)$. Data were analysed by one-way analysis of variance. Post hoc comparisons of means were carried out with Dunnett's post hoc test using GraphPad Prism software (ver. 6.1; San Diego, CA, USA). In all analyses, $p<0.05$ was taken to indicate statistical significance.

\section{Results}

\section{Icariin ameliorates the changes in seizure stage}

The effects of icariin on the seizure stages in the neonatal epilepsy rat model are shown in Figure 1. The seizure stage was increased to 3.8 in the hypoxia group compared to the normal group, but decreased to 1.5 in the icariin group.

\section{Icariin ameliorates the effects on cognitive function}

Cognitive function was examined in icariin-treated neonatal epilepsy rats using the Morris water maze test. The hypoxia group showed decreases in the number of crossings and percentage of time spent in the target quadrant and an increase in the escape latency time compared to the normal control group. However, treatment with icariin ameliorated the alterations in number of crossings, percentage of time spent in the target quadrant and escape latency time in neonatal epilepsy rats (Figure 2).

\section{Icariin ameliorates the effects on electroencephalography parameters}

The percentage of duration of seizures and percentage of duration of EEG ictal activity were examined in icariin-treated neonatal epilepsy rats (Figure 3). The icariin group showed decreases in the percentages of seizure duration and of EEG ictal activity compared to the hypoxia group.

\section{Icariin ameliorates oxidative stress}

Parameters of oxidative stress, such as level of lipid peroxidation and SOD activity, were examined in the brain tissue of icariin-treated neonatal epilepsy rats. The hypoxia group showed increased MDA level and reduced SOD activity in the wet brain tissue compared to the normal group. However, the changes in SOD activity and MDA level were ameliorated in the icariin group compared to the hypoxia group (Figure 4).

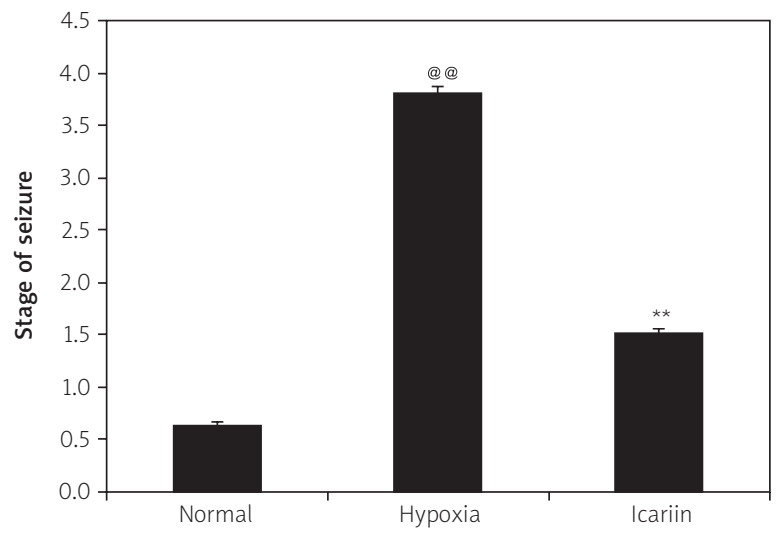

Fig. 1. Icariin ameliorates changes in seizure stages in the hypoxia-induced neonatal epilepsy rat model. Data are shown as the mean \pm SEM $(n=8)$. @ $p<0.01$ compared to the normal group, ${ }^{* *} p<0.01$ compared to the hypoxia group. 

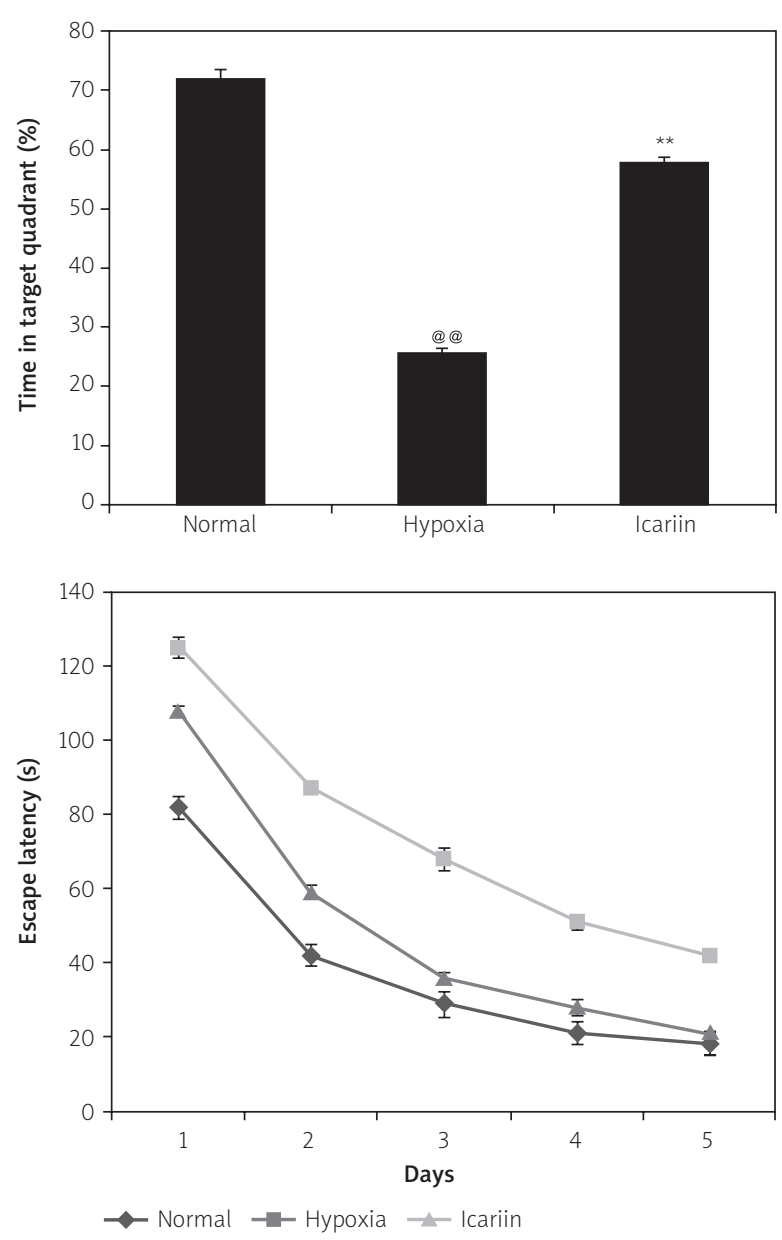

\section{Icariin ameliorates neuronal cell apoptosis}

The effects of icariin on the expression of apoptotic proteins in the brain tissue of hypoxia-induced epilepsy neonatal rats were examined. The expression of caspase- 3 and Bax protein were increased and the expression of $\mathrm{Bcl}-2$ protein was decreased in the tissue homogenate of the hypoxia group compared to the normal group. Treatment with icariin ameliorated these alterations in Bax, $\mathrm{Bcl}-2$ and caspase- 3 protein expression in the brain tissue homogenates of neonatal epilepsy rats (Figure 5).

\section{Icariin ameliorates the changes in GluR2, ERK I/II and $p$-ERK I/II protein expression}

Figure 6 shows the effects of icariin on GluR2, ERK I/II and p-ERK I/II protein expression in the brain tissue of the hypoxia-induced epilepsy neonatal rat

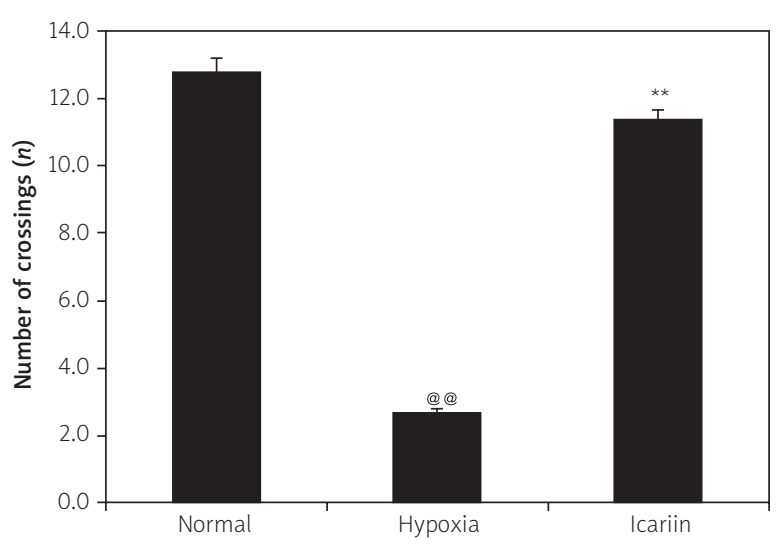

Fig. 2. Icariin ameliorates changes in cognitive function detected using the Morris water maze paradigm in the hypoxia-induced neonatal epilepsy rat model. Data are shown as the mean $\pm \operatorname{SEM}(n=8)$. $@ p<0.01$ compared to the normal group, ${ }^{* \star} p<0.01$ compared to the hypoxia group.

model. Expression of ERK I/II was comparable in all groups of rats examined in this study, while p-ERK I/II and GluR2 protein expression were increased in the brain tissue of the hypoxia group compared to the normal group. These changes in expression of $p$-ERK I/II and GluR2 protein were reduced in the icariin group compared to the hypoxia group.

\section{Icariin ameliorates neuronal injury}

The numbers of neurons in the brain tissue of the hypoxia-induced epilepsy neonatal rats were determined by Nissl staining (Figure 7). Histopathological analysis of the brain tissue showed morphological abnormalities, i.e. blurred caryotheca and pyknotic nuclei, in the hypoxia group. The density of surviving neurons in the brain tissue was reduced in the hypoxia group compared to the normal group. However, the icariin group showed amelioration of the neuronal damage in the brain tissue. 

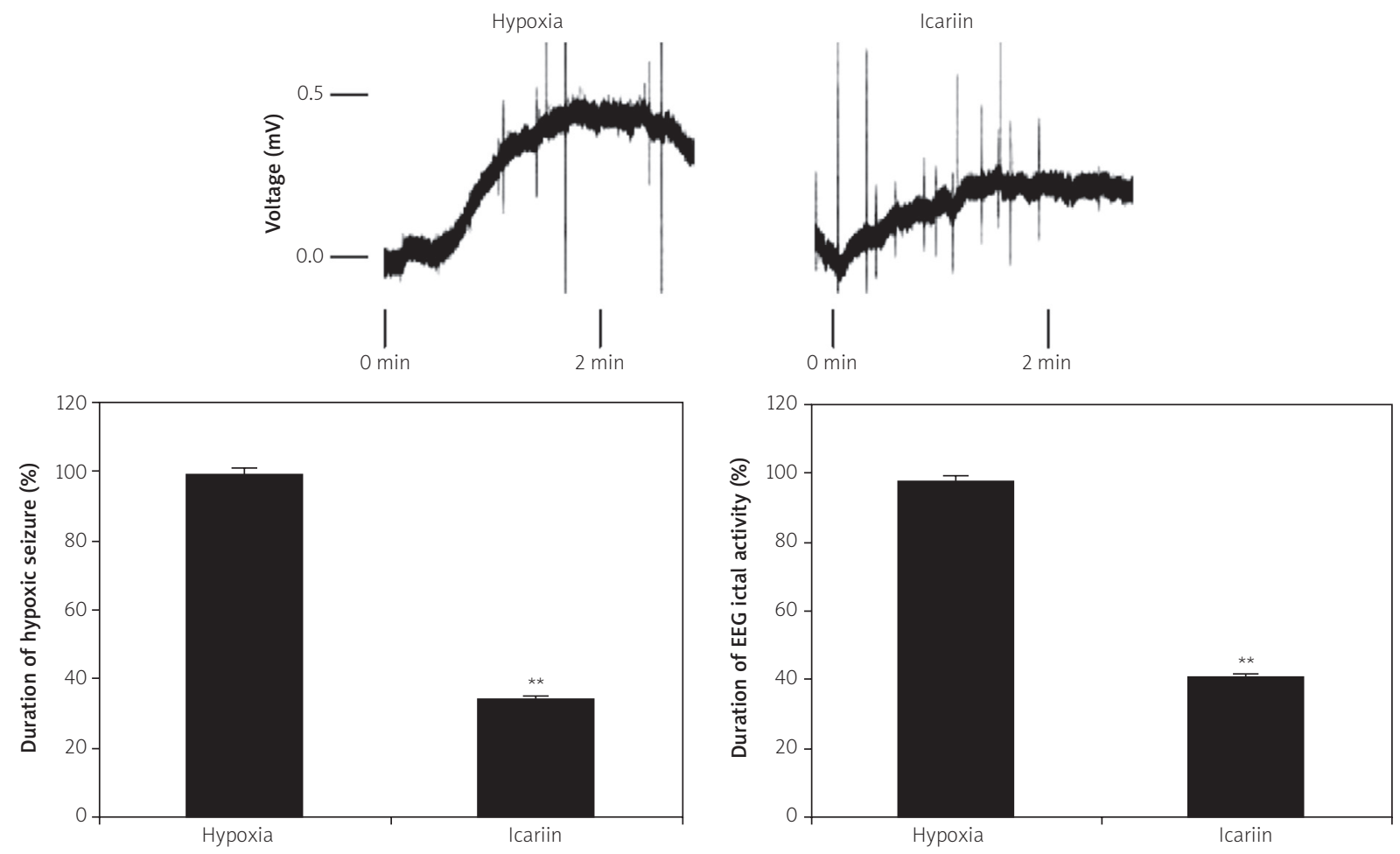

Fig. 3. Icariin ameliorates the duration of seizures and changes in EEG parameters in the hypoxia-induced neonatal epilepsy rat model. Data are shown as the mean \pm SEM $(n=8)$. @ $p<0.01$ compared to the normal group, ${ }^{* *} p<0.01$ compared to the hypoxia group.
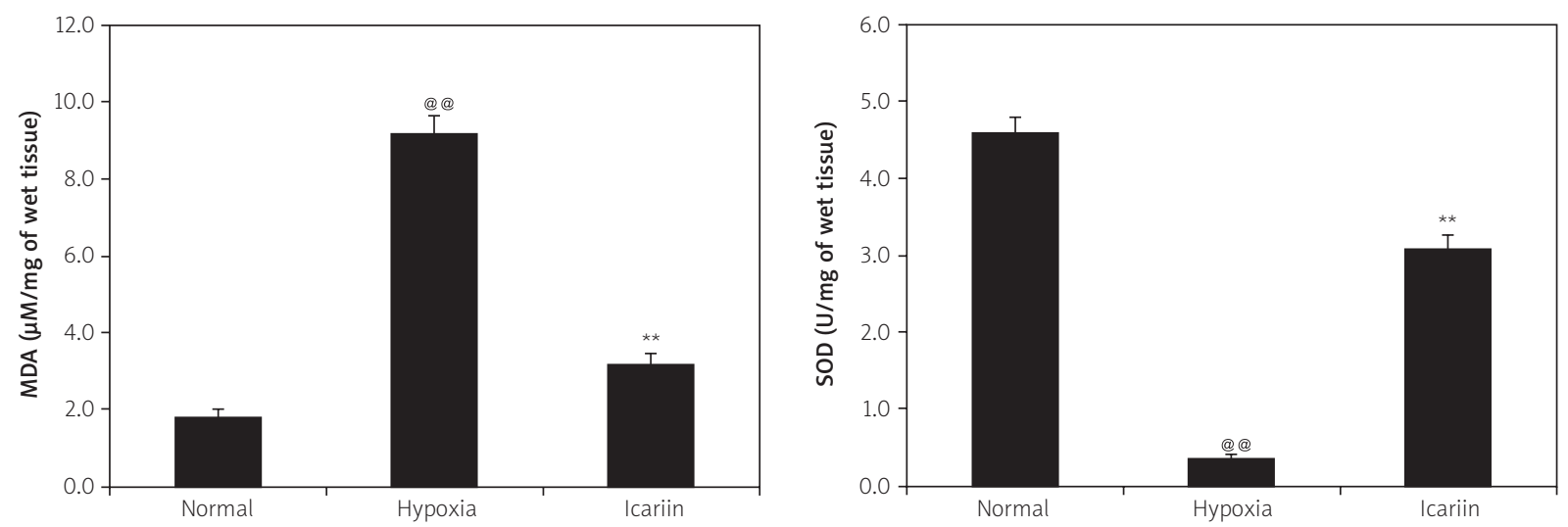

Fig. 4. Icariin ameliorates the changes in parameters of oxidative stress in the hypoxia-induced neonatal epilepsy rat model. Data are shown as the mean \pm SEM $(n=8)$. ${ }^{\circledR} p<0.01$ compared to the normal group, ${ }^{* *} p<0.01$ compared to the hypoxia group.

\section{Discussion}

Conventional anti-epilepsy drugs do not show beneficial effects in patients with neonatal epilepsy. The present study was performed to evaluate the protective effects of the flavonoid icariin, the main active compound isolated from plants belonging to the genus Epimedium, on cognitive function in a hypoxia-induced neonatal epilepsy rat model. The effects of icariin were examined by estimating seizure stages, cognitive function and EEG parameters in neonatal epilepsy rats. Parameters of oxidative stress and protein expression profiles were determined by histopathological analysis and Western 

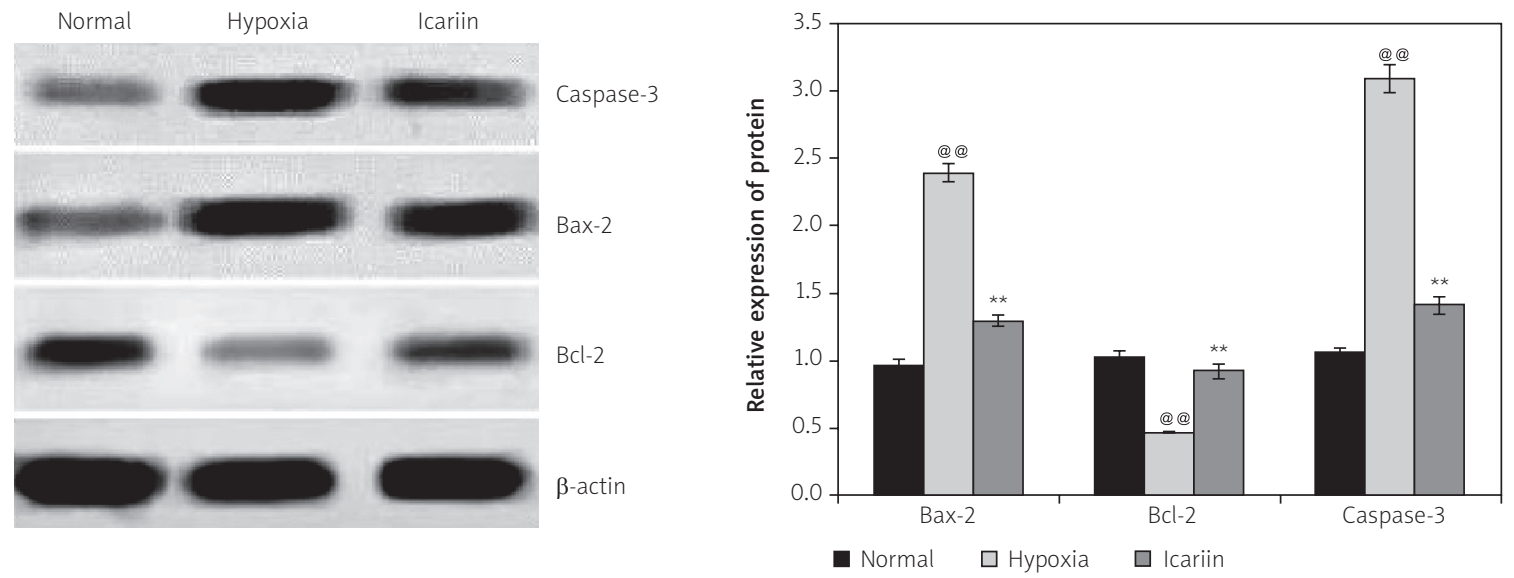

Fig. 5. Icariin ameliorates the expression of apoptosis proteins in brain tissue in the hypoxia-induced neonatal epilepsy rat model. Data are shown as the mean \pm SEM $(n=8)$. @@ $p<0.01$ compared to the normal group, ${ }^{* *} p<0.01$ compared to the hypoxia group.
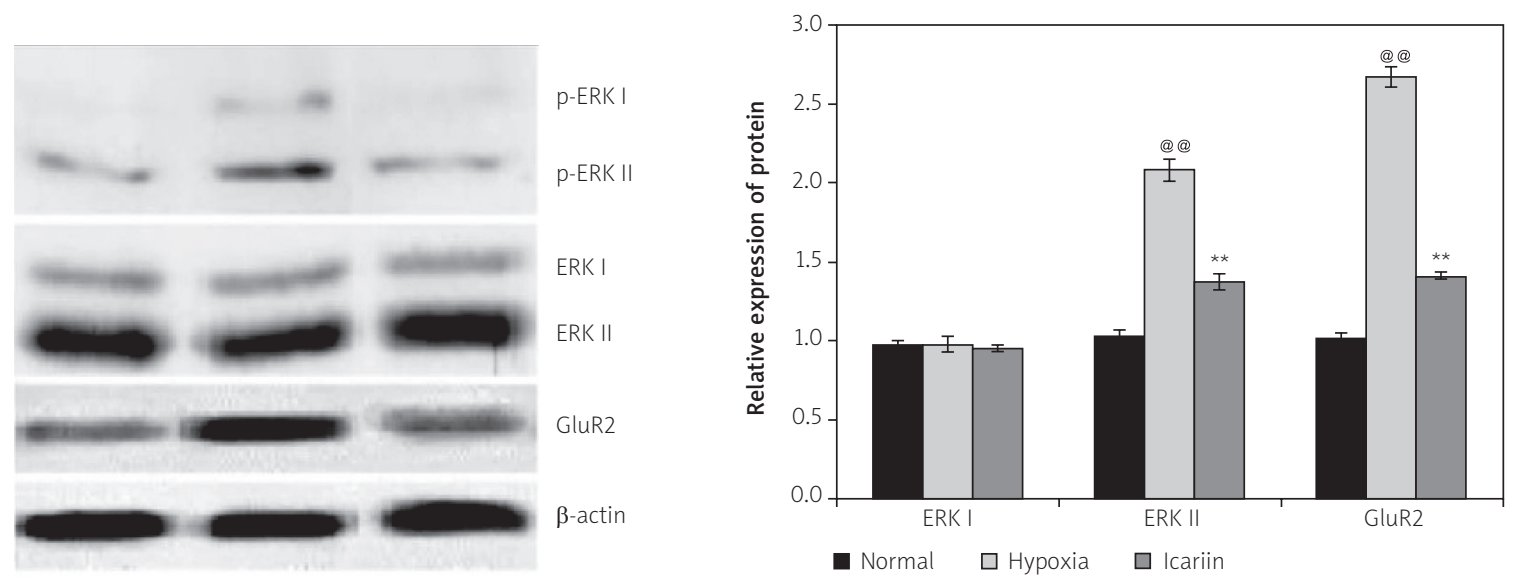

Fig. 6. Icariin ameliorates the changes in GluR2, ERK I/II and p-ERK I/II protein expression in brain tissue in the hypoxia-induced neonatal epilepsy rat model. Data are shown as the mean $\pm \operatorname{SEM}(n=8)$. ${ }^{\circledR} p<0.01$ compared to the normal group, ${ }^{\star *} p<0.01$ compared to the hypoxia group.
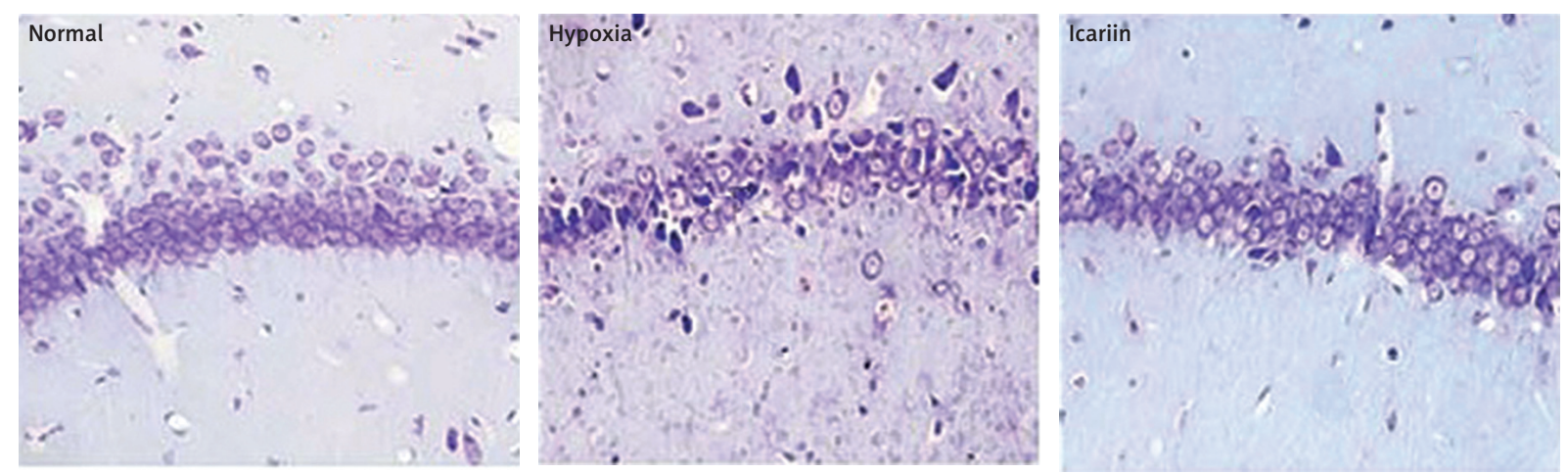

Fig. 7. Icariin ameliorates the changes in number of neurons in brain tissue in the hypoxia-induced neonatal epilepsy rat model. 
blotting in the brain tissue of the neonatal epilepsy rat model.

Perinatal asphyxia and hypoxia are the two major pathogenic factors contributing to the development of neonatal epilepsy [13]. Moreover, susceptibility to excitation for long periods of time is enhanced by hypoxia, and this also leads to development of neuronal injury [14]. The results of the present study indicated that hypoxia increases both the stage and number of seizures. Neuronal injury in seizure may contribute to alteration of cognitive function [23], which was supported by the results of the present study. The results presented here showed that treatment with icariin reduced the seizure stage and number in the hypoxia-induced seizure rat model. Moreover, cognitive function was recovered in the icariin treatment group compared to the hypoxia group.

Hypoxia increases the production of reactive oxygen species, thereby increasing oxidative stress [7]. Enhancement of oxidative stress alters the cellular activity, including neuronal cells function and impulse generation [3]. The results of the present study showed that oxidative stress was increased in the brain tissue of the hypoxia group and that treatment with icariin attenuated the level of oxidative stress in the brain tissue of hypoxia-induced neonatal epilepsy rats.

These changes cause imbalances in the levels of several neurotransmitters in the brain of rats exposed to hypoxia, and results in impairment of synaptic plasticity, downregulation of GABAergic inhibition and AMPA potentiation leading to modulation and enhancement of the sensitivity of brain tissue under hypoxic conditions [2]. GluR2 and p-ERK I/II contribute to the potentiation of AMPA [8]. The results of the present study suggested that treatment with icariin attenuated the alterations in GluR2 and $p$-ERK I/II protein expression in the brain tissue of hypoxia-induced neonatal epilepsy rats. Moreover, the histopathological changes in the brain tissue were ameliorated in the icariin treatment group compared to the hypoxia group.

\section{Conclusions}

The results of the present study suggest that icariin protects against neuronal injury and improves cognitive function in the hypoxia-induced neonatal epilepsy rat model by modulating the GluR2/ERK I/ II pathway.

\section{Acknowledgements}

The authors are grateful to Northwest Women's and Children's Hospital, Xi'an, China, for providing all of the necessary facilities to conduct this study.

\section{Disclosure}

The authors declare no conflict of interest.

\section{References}

1. Behuet S, Cremer JN, Cremer M, Palomero-Gallagher N, Zilles K, Amunts K. Developmental changes of glutamate and GABA receptor densities in Wistar rats. Front Neuroanat 2019; 13: 100.

2. Deidda G, Bozarth IF, Cancedda L. Modulation of GABAergic transmission in development and neurodevelopmental disorders: investigating physiology and pathology to gain therapeutic perspectives. Front Cell Neurosci 2014; 8: 119.

3. Di Meo S, Reed TT, Venditti P, Victor VM. Role of ROS and RNS sources in physiological and pathological conditions. Oxid Med Cell Longev 2016; 2016: 1245049.

4. Ding L, Liang XG, Zhu DY, Lou YJ. Icariin promotes expression of PGC-1alpha, PPARalpha, and NRF-1 during cardiomyocyte differentiation of murine embryonic stem cells in vitro. Acta Pharmacol Sin 2007; 28: 1541-1549.

5. El-Shitany NA, Eid BG. Icariin modulates carrageenan-induced acute inflammation through $\mathrm{HO}-1 / \mathrm{Nrf2}$ and NF-kB signaling pathways. Biomed Pharmacother 2019; 120: 109567.

6. Glass HC, Hong KJ, Rogers EE, Jeremy RJ, Bonifacio SL, Sullivan JE, Barkovich AJ, Ferriero DM. Risk factors for epilepsy in children with neonatal encephalopathy. Pediatr Res 2011; 70 : 535-540.

7. Görlach A, Dimova EY, Petry A, Martínez-Ruiz A, HernansanzAgustín P, Rolo AP, Palmeira CM, Kietzmann T. Reactive oxygen species, nutrition, hypoxia and diseases: Problems solved? Redox Biol 2015; 6: 372-385.

8. Henley JM, Wilkinson KA. AMPA receptor trafficking and the mechanisms underlying synaptic plasticity and cognitive aging. Dialogues Clin Neurosci 2013; 15: 11-27.

9. Holmes GL. Effect of seizures on the developing brain and cognition. Semin Pediatr Neurol 2016; 23: 120-126.

10. lihara K, Joo DT, Henderson J, Sattler R, Taverna FA, Lourensen S, Orser BA, Roder JC, Tymianski M. The influence of glutamate receptor 2 expression on excitotoxicity in Glur2 null mutant mice. J Neurosci 2001; 21: 2224-2239.

11. Kasahara Y, Ikegaya Y, Koyama R. Neonatal seizure models to study epileptogenesis. Front Pharmacol 2018; 9: 385.

12. Li L, Peng L, Miao J, Qiu Y, Zhou Y, Gao X, Xu Y, Shi Z, Shao D, Ma Z. Icariin induces the expression of toll-like receptor 9 in ana-1 murine macrophages. Phytother Res 2011; 25: 1732-1735.

13. Millar LJ, Shi L, Hoerder-Suabedissen A, Molnár Z. Neonatal hypoxia ischaemia: mechanisms, models, and therapeutic challenges. Front Cell Neurosci 2017; 11: 78.

14. Mukandala G, Tynan R, Lanigan S, O'Connor JJ. The effects of hypoxia and inflammation on synaptic signaling in the CNS. Brain Sci 2016; 6: 6 . 
15. Qin L, Han T, Zhang Q, Cao D, Nian H, Rahman K, Zheng H. Antiosteoporotic chemical constituents from Er-Xian Decoction, a traditional Chinese herbal formula. J Ethnopharmacol 2008; 118: 271-279.

16. Sanchez RM, Koh S, Rio C, Wang C, Lamperti ED, Sharma D, Corfas G, Jensen FE. Decreased glutamate receptor 2 expression and enhanced epileptogenesis in immature rat hippocampus after perinatal hypoxia-induced seizures. J Neurosci 2001; 21: 8154-8163.

17. Scharfman HE. The neurobiology of epilepsy. Curr Neurol Neurosci Rep 2007; 7: 348-354.

18. Schidlitzki A, Twele F, Klee R, Waltl I, Römermann K, Bröer S, Meller S, Gerhauser I, Rankovic V, Li D, Brandt C, Bankstahl M, Töllner K, Löscher W. A combination of NMDA and AMPA receptor antagonists retards granule cell dispersion and epileptogenesis in a model of acquired epilepsy. Sci Rep 2017; 7: 12191.

19. Shen R, Wang JH. The effect of icariin on immunity and its potential application. Am J Clin Exp Immunol 2018; 7: 50-56.

20. Vorhees CV, Williams MT. Morris water maze: procedures for assessing spatial and related forms of learning and memory. Nat Protoc 2006; 1: 848-858.

21. Wang C, Wang X, Xu L, Cheng Y. Neuroprotective activity of icariin against hypoxic-ischemic brain injury in neonatal rats. Int J Pharmacol 2019; 15: 829-836.

22. Wang GQ, Li DD, Huang C, Lu DS, Zhang C, Zhou SY, Liu J, Zhang F. Icariin reduces dopaminergic neuronal loss and microglia-mediated inflammation in vivo and in vitro. Front Mol Neurosci 2018; 10: 441.

23. Wu Q, Zhao CW, Long Z, Xiao B, Feng L. Anatomy based networks and topology alteration in seizure-related cognitive outcomes. Front Neuroanat 2018; 12: 25.

24. Xu CQ, Liu BJ, Wu JF, Xu YC, Duan XH, Cao YX, Dong JC. Icariin attenuates LPS-induced acute inflammatory responses: involvement of PI3K/Akt and NF-kappaB signaling pathway. Eur J Pharmacol 2010; 642: 146-153. 